\title{
Evolution of the $N=20$ and 28 shell gaps and two-particle-two-hole states in the FSU interaction
}

\author{
R. S. Lubna $\odot,{ }^{1, *}$ K. Kravvaris, ${ }^{1,2}$ S. L. Tabor, ${ }^{1}$ Vandana Tripathi,,${ }^{1}$ E. Rubino $\odot,{ }^{1}$ and A. Volya ${ }^{1}$ \\ ${ }^{1}$ Department of Physics, Florida State University, Tallahassee, Florida 32306, USA \\ ${ }^{2}$ Lawrence Livermore National Laboratory, Livermore, California 94550, USA
}

(Received 23 February 2020; revised 21 October 2020; accepted 18 November 2020; published 8 December 2020)

\begin{abstract}
The connection between fundamental nucleon-nucleon forces and the observed many-body structure of nuclei is a main question of modern nuclear physics. Evolution of the mean field, inversion of traditional shell structures, and structure of high spin states in nuclei with extreme proton-to-neutron ratios are at the center of numerous recent experimental investigations targeting the matrix elements of the effective nuclear Hamiltonian that is responsible for these phenomena. The FSU spsdf $p$ cross-shell interaction for the shell model was successfully fitted to a wide range of mostly intruder negative parity states of the $s d$ shell nuclei. In this paper, we explore the evolution of nuclear structure in and around the island of inversion (IoI), where low-lying states involve crossshell particle-hole excitations. We apply the FSU interaction to systematically trace out the relative positions of the effective single-particle energies of the $0 f_{7 / 2}$ and $1 p_{3 / 2}$ orbitals forming the $N=20$ and 28 shell gaps. We find that above a proton number of about 13 , the $0 f_{7 / 2}$ neutron orbital lies below that of $1 p_{3 / 2}$, which is considered normal ordering but, systematically, for more exotic nuclei with lower $Z=12$ and 10 the order of orbitals reversed. The crossing of the neutron orbitals happens right near the neutron separation threshold. Our Hamiltonian reproduces remarkably well the absolute binding energies for a broad range of nuclei and the inversion in the configurations of nuclei inside the IoI. The effective interaction accounts well for the energies and variations with mass number $A$ of aligned high-spin states that involve nucleon pairs prompted across the shell gap. This paper puts forward an empirically determined effective Hamiltonian where data from many recent experiments allowed us to significantly improve our knowledge about cross-shell nuclear interaction matrix elements. The quality with which this Hamiltonian describes the two-particle, two-hole cross-shell excitations, binding energies, and the physics of aligned states that were not a part of the fit, is remarkable, making the FSU interaction an important tool for the future exploration of exotic nuclei.
\end{abstract}

DOI: 10.1103/PhysRevResearch.2.043342

\section{INTRODUCTION}

Recent experimental works in the $1 s 0 d$ shell with large $\gamma$ detector arrays and heavy-ion fusion reactions have substantially extended the knowledge of relatively high spin states. However, these do not form well-behaved rotational bands amenable to study by collective models because rotational energies are comparable to single-particle energies (SPEs). On the other hand, microscopic configuration-interaction model calculations are feasible in these lighter nuclei. The USD family of effective interactions [1,2] have been very successful in describing most lower-lying positive-parity states of nuclei with $8 \leqslant(N, Z) \leqslant 20$. However, higher spin states involve excitations into the $f p$ shell where orbitals contributing larger values of angular momentum are occupied, which is beyond

\footnotetext{
*Present address: TRIUMF, Vancouver, BC V6T 2A3, Canada; rebeka.sultana.00@gmail.com

Published by the American Physical Society under the terms of the Creative Commons Attribution 4.0 International license. Further distribution of this work must maintain attribution to the author(s) and the published article's title, journal citation, and DOI.
}

the scope of the USD interaction. Also, neutron-rich isotopes quickly move beyond the $s d$ shell boundaries [3-8].

Over the years, several configuration interaction models have made significant contributions toward explaining crossshell excitations [9-13]. A case in point is the island of inversion (IoI). Perhaps in an inverse way, the first contribution came from the failure of the otherwise very successful pure $s d$ interactions $[1,2]$ to reproduce the stronger binding energy measured for ${ }^{31} \mathrm{Na}$ [14], pointing to the importance of effects outside the $s d$ shell. The WBMB [15] interaction, which was designed for the nuclei near ${ }^{40} \mathrm{Ca}$, was successful in reproducing the inversion of some nuclei within the IoI. More recent shell-model calculations using interactions like SDPF-M [12] and SDPF-U-MIX [13] have shown that the IoI phenomenon can be accounted for by a reduction of the $N=20$ shell gap. Recently, a significant theoretical result was reported, see Ref. [16], showing the emergence of the IoI effect from nucleon-nucleon forces stemming from the fundamental principles of QCD. This highlights the importance of certain cross $s d-f p$ interaction terms that we assess in this paper using experimental systematics.

In searching for a single cross-shell interaction which works well over a wide range of nuclei, we have developed an interaction [17] with parallel treatment of protons and neutrons by fitting the energies of 270 states in nuclei from 
${ }^{13} \mathrm{C}$ through ${ }^{51} \mathrm{Ti}$ and ${ }^{49} \mathrm{~V}$ originated from the WBP interaction [18] using well-established techniques. The present paper is organized as follows: First, we will discuss the development of the FSU shell-model interaction. The trend of the effective single-particle energies (ESPEs) of the $0 f_{7 / 2}$ and $1 p_{3 / 2}$ orbitals for the $s d$-shell nuclei will be examined along with a comparison to the experimental data. Then we will move to the IoI region and test some predictions of the FSU interaction in this region. Finally, the experimentally observed fully aligned states with the $f_{7 / 2}^{2}$ configuration will be interpreted with the FSU shell-model interaction.

\section{DEVELOPMENT OF THE FSU EMPIRICAL SHELL-MODEL INTERACTION}

A modified version of the WBP [18] interaction has been used as a starting point of the data fitting procedure. The WBP interaction was developed in order to address the cross-shell structure around $A=20$. While the $s d-f p$ cross-shell matrix elements of the WBP were taken from the WBMB [15] interaction, which was developed for the nuclei around ${ }^{40} \mathrm{Ca}$, the different SPEs and different implementation make WBP unsuitable for the upper $s d$-shell nuclei. Yet, the WBP is a perfect starting point for a more modern, much broader assessment of the nuclear matrix elements. Our data set included nuclei from the upper mass region of the $p$, the full $s d$, and lower mass region of the $f p$ shells; where we systematically looked at states that involve a particle promotion across the harmonic oscillator shell, referred to as one-particle, one-hole excited states $(1 \mathrm{p} 1 \mathrm{~h})$. In the $s d$-shell region of most interest and most data, the combined $0 \mathrm{p} 0 \mathrm{~h}$ and $1 \mathrm{p} 1 \mathrm{~h}$ space considered in the fit is equivalent to $0 \hbar \omega$ and $1 \hbar \omega$, often referred to as the $N_{\max }=1$ harmonic oscillator basis truncation. The resulting fit seamlessly spans from the $A=20$ region, where the lowlying intruder states are predominantly those with holes in the lower $p$ shell, to the IoI around $A=40$ where particles are promoted to $f p$ shell. The ability to separate the center of mass exactly within the $N_{\max }=1$ harmonic oscillator basis truncation is an additional benefit of this strategy.

Before the current effort of developing the FSU interaction, a number of attempts have been made to modify the WBP interaction, mainly by changing the SPEs of the $f p$-shell orbitals for a particular $s d$-shell nucleus and applying it for the nearby isotopes. For example, in the WBP-A [19] version, the SPEs of the $f_{7 / 2}$ and $p_{3 / 2}$ orbitals were lowered in order to better explain the negative parity intruder states of ${ }^{34} \mathrm{P}$. This adjustment was quite successful in explaining the energy levels of ${ }^{32} \mathrm{P}$ and ${ }^{36} \mathrm{P}$, however, WBP-A failed to predict the intruder states of ${ }^{31} \mathrm{Si}$. Hence, another version of the WBP, called WBP-B, was introduced [20] by changing the SPEs of the $f_{7 / 2}, p_{3 / 2}$, and $p_{1 / 2}$ orbitals. In a different version, named WBP-M [21], all the SPEs of the $f p$ shell orbitals were changed in order to reproduce the energies and the ordering of the $3 / 2^{-}$and $7 / 2^{-}$states of ${ }^{27} \mathrm{Ne}$ which eventually fixed the ordering of the same levels in ${ }^{25} \mathrm{Ne}$ and ${ }^{29} \mathrm{Mg}$. However, none of these modified versions were able to reproduce the experimental data for a large range of the nearby nuclei, and hence we have taken a step forward toward building a more general effective shell-model Hamiltonian.
The model space for the WBP interaction, and for this one, consists of four major oscillator shells: $0 s, 0 p, 1 s 0 d$, and $0 f 1 p$. The following steps briefly describe the development of the FSU interaction:

(1) The interaction starts from the WBP framework; the model consists of four major oscillator shells: $0 s, 0 p, 1 s 0 d$, and $0 f 1 p$. Isospin invariance is assumed and Coulomb corrections to the binding energies are implemented using the standard procedures as discussed in Refs. $[1,2,15]$.

(2) The SPEs and the two-body matrix elements (TBMEs) of the $0 s$ and $0 p$ shells and across $0 s-0 p$ are same as those of the original WBP interaction and are not a part of the fit.

(3) The TBMEs within the $s d$ shell are taken from the USDB [2] interaction and also are not part of the fit.

(4) The six monopoles between the orbitals of the $0 p$ shell and $s d$ shell are modified simultaneously with the $s d$ shell SPEs, thus changing the shell gap but ensuring that excitation energies of all $0 \hbar \omega$ states in the $s d$ shell are identical to those from the USDB calculations.

(5) $s d-f p$ cross-shell matrix elements:

(a) The $1 p_{1 / 2}$ orbital in the $f p$ shell is relatively high and not very sensitive to our data set. We thus fitted only one monopole term between the $1 p_{1 / 2}$ and $s d$ orbitals. This amounts to two fit parameters because we have allowed different strengths for isospins $T=0$ and $T=1$.

(b) Only the monopole terms between $0 f_{7 / 2}-0 d_{5 / 2}$ and $1 p_{3 / 2}-0 d_{5 / 2}$ were considered since $d_{5 / 2}$ is deeply bound for $s d-f p$ cross-shell nuclei. A total of four parameters were varied for $T=0$ and $T=1$.

(c) For the remaining $0 f_{7 / 2}-1 s_{1 / 2}, \quad 0 f_{7 / 2}-0 d_{3 / 2}$, $1 p_{3 / 2}-0 s_{1 / 2}$, and $1 p_{3 / 2}-0 d_{3 / 2}$, all multipole-multipole density terms were fitted. A total of 24 parameters were varied.

(6) For the $f p$ shell, GXPF1A [22] was used as a starting Hamiltonian and all the TBMEs associated with only $0 f_{7 / 2}$ and $1 p_{3 / 2}$ were fitted; a total 30 TBMEs and hence 30 parameters were adjusted within the $f p$ shell.

(7) All the matrix elements within the $s d$ and $f p$ shells as well as the $s d-f p$ cross shell were scaled with $A^{-0.3}$. However, no scaling was adopted for the cross-shell interactions between the lower $p$ and the $s d$ shells.

(8) A total of 70 parameters were fitted using 270 experimentally observed states compiled in Refs. [23,24]. The experimental data was compiled from four groups:

(a) Intruder states sensitive to $p$-sd shell gap. This group consists of pure $p$ shell $\mathrm{C}$ and $\mathrm{N}$ isotopes and nuclei between $\mathrm{O}$ to $\mathrm{Si}$ with states that have strong spectroscopic factors (SFs) if populated via $(p, d)$ reactions.

(b) Negative parity states in $s d$ shells populated via $(d, p)$ reactions which are sensitive to particle promotion from $s d$ to $f p$. High spin states that gain spin from the promotion of a particle to $0 f_{7 / 2}$ are of particular importance.

(c) Neutron-rich cross-shell nuclei with $Z<20$ and $N>20$, where both $0 \hbar \omega$ and $1 \hbar \omega$ types of states were included in the fit.

(d) Nuclei in $f p$ shells with $Z \geqslant 20$ and $N \geqslant 21$; the $0 \hbar \omega$ states in these nuclei are critical for tuning the $0 f_{7 / 2}-1 p_{3 / 2}$ gap. 


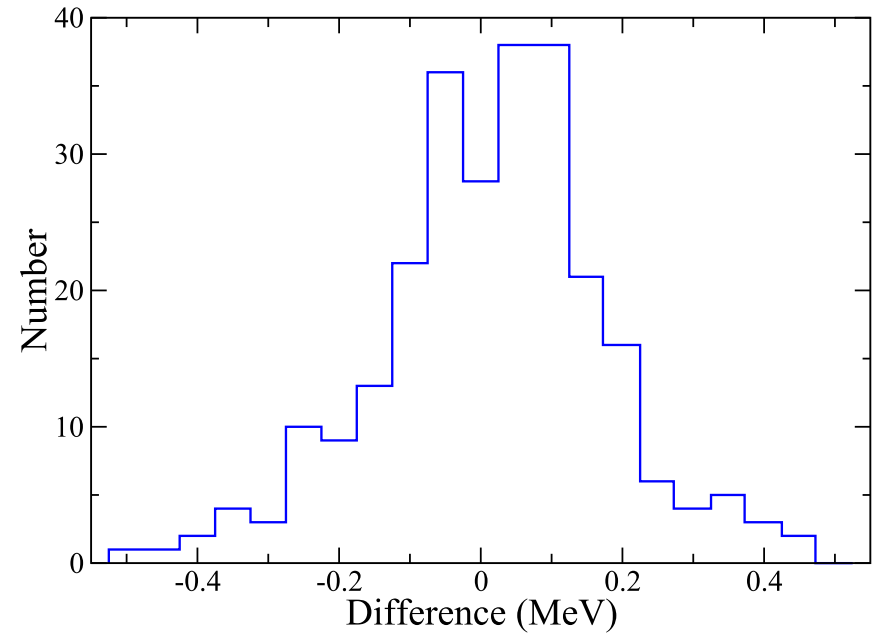

FIG. 1. Histogram of the differences in excitation energy between experiment and the FSU interaction fit. The root-mean-square deviation is $190 \mathrm{keV}$.

(9) The fitting procedure followed the method described in Ref. [2], with 40 linear combinations of parameters being selected at each iteration. We reached the convergence after six iterations with an overall $\mathrm{rms}$ deviation from experiment of $190 \mathrm{keV}$.

(10) All calculations were carried out within $N_{\max }=1$ truncation, thus including $0 \hbar \omega$ and $1 \hbar \omega$ types of excitations that, due to different parities, do not mix. This truncation allows for exact identification and separation of the spurious center-of-mass excitations.

(11) Tables of the matrix elements can be found in the thesis publication of Lubna [23]. Users are encouraged to contact the authors for help with the calculations, further details, and updates.

All the shell-model calculations were performed with the shell-model code CoSMo [25]. A histogram of the differences between the experimental states included in the fit and those predicted with the FSU interaction is shown in Fig. 1.

\section{EFFECTIVE SINGLE-PARTICLE ENERGY}

The evolution of the mean field, which is described by the position of the single-particle levels and how they change with the number of protons and neutrons, is a particularly interesting and nontrivial question in the strongly interacting two-component many-body systems of atomic nuclei. In most nuclei, the single-particle strength is distributed over many states. Systematic studies have been performed before with other shell-model interactions $[10,12,26]$ to understand the evolution of the ESPE. An experimental approach of determining the ESPEs has been to measure and sum up the energies of appropriate states weighted by the reaction SFs. This process is limited by decreasing cross sections for higher lying states and by difficulties in making spin assignments and in determining what fraction of the cross sections come from direct reaction components.

Theoretical approaches do not suffer from most of these experimental limitations, but have their own uncertainties. Perhaps chief among them being the uncertainty in the in-
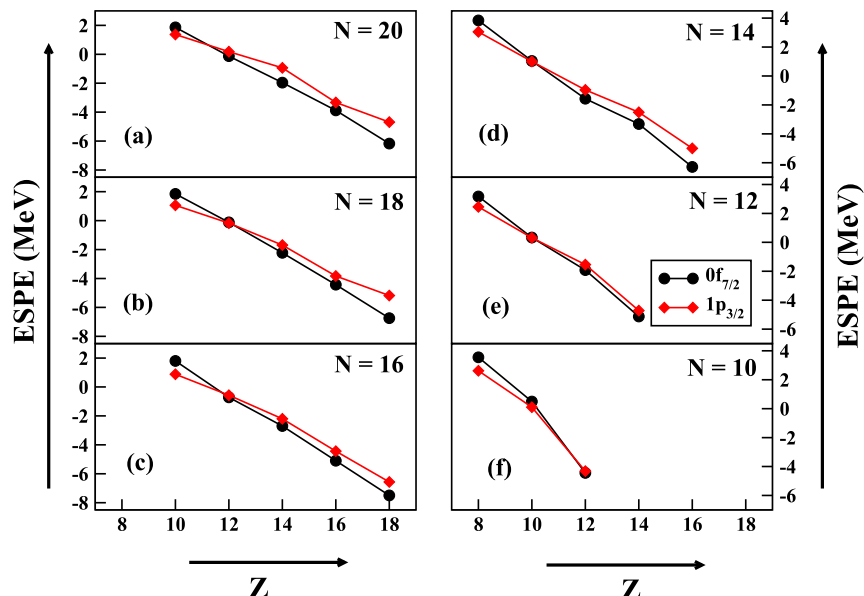

FIG. 2. Neutron effective single particle energies (ESPEs) of $0 f_{7 / 2}$ and $1 p_{3 / 2}$ orbitals calculated with the FSU interaction. They represent the theoretical centroids of the energies of the $0 f_{7 / 2}$ and $1 p_{3 / 2}$ orbitals. In the "normal" ordering, the red diamonds $\left(1 p_{3 / 2}\right)$ lie above the black circles $\left(0 f_{7 / 2}\right)$.

teraction Hamiltonian. The bare SPEs tell only a part of the story of the effective shell positions. The TBMEs have a major influence on the positions of the orbitals. In fact, the TBMEs shift the orbitals based on the number of particles in shells and are the major reason that one interaction could fit such a wide range of nuclei.

How this FSU interaction describes the shell evolution is among the most interesting immediate questions that can be addressed. While the FSU interaction was fitted to the negative-party states in $s d$ nuclei, the study of the ESPE extrapolates to a much broader spectrum of configurations not limited by those experimentally reachable with single-nucleon transfer reactions.

The evaluation of the ESPE relies on $0 \hbar \omega$ and $1 \hbar \omega$ calculations. In order to determine the ESPE of the $0 f_{7 / 2}$ and $1 p_{3 / 2}$ orbitals, we have followed a procedure similar to the experimental approach but using the theoretically computed energies and SFs in the following formula:

$$
\mathrm{ESPE}=\frac{\sum_{\mathrm{i}=1} \mathrm{SF}_{\mathrm{i}} \times \mathrm{E}_{\mathrm{i}}^{*}}{\sum_{\mathrm{i}=1} \mathrm{SF}_{\mathrm{i}}} .
$$

In the above formula, $\mathrm{SF}_{\mathrm{i}}$ is the $\mathrm{SF}$ for $A \rightarrow A+1$ where a particle is placed onto a single-particle orbit of interest above an even-even $A$ core. The $\mathrm{E}_{\mathrm{i}}^{*}$ is the excitation energy of the $i$ th state in $A+1$ with the matching quantum numbers measured relative to the ground-state energy of the core $A$. It has been observed from the calculations that it is enough to consider the 30 lowest states in the sum Eq. (1); by then, the SF reach a saturation and the ESPE converges. From the formal theoretical perspective, Eq. (1) represents SPEs of the mean field arising from the shell-model Hamiltonian.

The ESPEs obtained from the above formula across the $s d$ shell are plotted in Fig. 2 as a function of proton number $Z$. The points represent the ESPEs of the $0 f_{7 / 2}$ and $1 p_{3 / 2}$. The systematic crossing of the ESPEs of the $0 f_{7 / 2}$ and $1 p_{3 / 2}$ orbitals with increasing neutron number is evident in the figure. The crossing occurs between $Z=10$ and 12 , suggesting that 
the $N=28$ shell gap shifts to $N=24$ with lower $Z$, which points to the inversion of $0 f_{7 / 2}$ and $1 p_{3 / 2}$ neutron orbitals. The ground state of ${ }^{31} \mathrm{Ne}$ is tentatively assigned $3 / 2^{-}$as is the first excited state in ${ }^{27} \mathrm{Ne}[24]$. In ${ }^{27} \mathrm{Mg}$, the lowest $3 / 2^{-}$and $7 / 2^{-}$ states are essentially degenerate [24].

This inversion of the $1 p_{3 / 2}$ and $0 f_{7 / 2}$ ESPE is related to the two-body interactions between nucleons in the $s d$ and $f p$ shells; the effect of this interaction is density dependent and varies as a function of the shell fillings. In the FSU interaction, these TBMEs emerge as a consequence of fitting the energies of the states in a wide range of nuclei. Over half a century ago, Talmi and Unna [27] attributed the inversion of the $1 s_{1 / 2}$ and $0 p_{1 / 2}$ orbitals to the same principle. Alternate explanations, especially for the $1 s_{1 / 2}$ and $0 p_{1 / 2}$ cases, have been given in terms of the effects of weak binding on the mean field of low $\ell$ orbitals. Hoffman et al. [28] explored the weak binding effect for pure single-particle shells in a Woods-Saxon potential and have shown that it is large near the threshold for neutron $s$ states. While much smaller for $p$ states, there is still a crossing between the $0 p_{1 / 2}$ and $0 d_{5 / 2}$ orbitals at the threshold. A similar effect for $1 p_{3 / 2}$ and $0 f_{7 / 2}$ appears to be a contributing factor to the inversion shown in Fig. 2. Nearly all crossings occur around $\mathrm{ESPE}=0$, indicating that the levels become unbound. Indeed, the centrifugal barrier for $\ell=3 f$ orbitals is high, which would make a transition into the continuum smooth, while for the $\ell=1 p$-wave, the interaction with the continuum is strong and is pushing the level down, as discussed in Ref. [29]. It appears that the continuum effect is incorporated through the fitting of the effective interaction, but this can be a challenge for theoretical methods that do not take continuum of reaction states into account. This inversion of the $1 p_{3 / 2}$ and $0 f_{7 / 2}$ ESPE at high neutron excess also has implications for the IoI phenomenon discussed in the next section.

Another way of examining the systematics of shell evolution, which is closer to experiments, is from the positions of the states carrying the largest part of the single-particle strength. Such a comparison is shown in Table I, which lists the experimental and theoretical excitation energies of the lowest $3 / 2^{+}, 7 / 2^{-}$, and $3 / 2^{-}$states, of the even- $Z$, odd-mass nuclei, along with the predicted and measured $(d, p)$ reaction SFs. As mentioned before, there is more uncertainty in measuring the values of SFs than excitation energies and in some cases the SFs cannot (lack for appropriate targets) or have not been measured. With this in mind, the agreement between experiments and predictions using the FSU interaction for both excitation energies and SFs is generally good. Also, the relatively large values of the SFs show that these states represent the dominant single-particle states.

Figure 3(a) provides a pictorial summary of the relative positions between the $7 / 2^{-}$and $3 / 2^{-}$states as a function of the proton number $Z$. The black circles and red lines show the average values from Table I for experiment and theory, respectively, while the black error bars represent the variation of the experimental differences. The observed trends are reproduced by theory, see Fig. 3(a). This graph agrees qualitatively with those in Fig. 2 . It demonstrates that the evolution of the separation between the $7 / 2^{-}$and $3 / 2^{-}$states is largely a function of the proton number $Z$ and that the $3 / 2^{-}$ energies drop below the $7 / 2^{-}$ones between $Z=14$ and 12 . In contrast to the ESPEs which approximate the positions of
TABLE I. Comparison of the experimentally observed $7 / 2^{-}$, $3 / 2^{-}$, and $3 / 2^{+}$states of even- $Z$, odd-mass $s d$-shell nuclei to the predictions by the FSU interaction. The measured spectroscopic factors were taken from the NNDC [24]. All the experimental spectroscopic factors were compiled from the $(d, p)$ reactions.

\begin{tabular}{|c|c|c|c|c|c|}
\hline \multirow[b]{2}{*}{ Nucleus } & \multirow[b]{2}{*}{$\mathbf{J}^{\pi}$} & \multicolumn{2}{|c|}{ Energy } & \multicolumn{2}{|c|}{$(2 J+1) S F$} \\
\hline & & EXP & Th & EXP & $\mathrm{Th}$ \\
\hline \multirow[t]{3}{*}{${ }^{25} \mathrm{Ne}$} & $7 / 2^{-}$ & 4030 & 3957 & 5.8 & 4.5 \\
\hline & $3 / 2^{-}$ & 3330 & 3471 & 3.0 & 1.9 \\
\hline & $3 / 2^{+}$ & 2030 & 2044 & 1.6 & 1.8 \\
\hline \multirow[t]{3}{*}{${ }^{27} \mathrm{Ne}$} & $7 / 2^{-}$ & 1740 & 1634 & 2.8 & 3.9 \\
\hline & $3 / 2^{-}$ & 765 & 858 & 2.6 & 2.4 \\
\hline & $3 / 2^{+}$ & 0 & 0 & 1.7 & 2.8 \\
\hline \multirow[t]{3}{*}{${ }^{25} \mathrm{Mg}$} & $7 / 2^{-}$ & 3971 & 3902 & $2.2-3.3$ & 3.9 \\
\hline & $3 / 2^{-}$ & 3413 & 3525 & $0.9-1.2$ & 1.5 \\
\hline & $3 / 2+$ & 974 & 1098 & 0.8 & 0.9 \\
\hline \multirow[t]{3}{*}{${ }^{27} \mathrm{Mg}$} & $7 / 2^{-}$ & 3761 & 3827 & 4.6 & 3.5 \\
\hline & $3 / 2^{-}$ & 3559 & 3644 & 1.6 & 2.2 \\
\hline & $3 / 2+$ & 984 & 994 & 2.4 & 1.56 \\
\hline \multirow[t]{3}{*}{${ }^{29} \mathrm{Mg}$} & $7 / 2^{-}$ & 1430 & 1719 & 3.0 & 4.4 \\
\hline & $3 / 2^{-}$ & 1094 & 1396 & 0.4 & 2.0 \\
\hline & $3 / 2^{+}$ & 0 & 0 & 1.2 & 1.8 \\
\hline \multirow[t]{3}{*}{${ }^{29} \mathrm{Si}$} & $7 / 2^{-}$ & 3623 & 3684 & 7.0 & 4.5 \\
\hline & $3 / 2^{-}$ & 4934 & 4373 & 2.2 & 2.3 \\
\hline & $3 / 2^{+}$ & 1273 & 1285 & 3.0 & 2.7 \\
\hline \multirow[t]{3}{*}{${ }^{31} \mathrm{Si}$} & $7 / 2^{-}$ & 3134 & 2855 & 4.8 & 5.6 \\
\hline & $3 / 2^{-}$ & 3533 & 3435 & 1.6 & 2.8 \\
\hline & $3 / 2^{+}$ & 0 & 0 & 2.8 & 2.4 \\
\hline \multirow[t]{3}{*}{${ }^{33} \mathrm{Si}$} & $7 / 2^{-}$ & 1435 & 1452 & & 6.0 \\
\hline & $3 / 2^{-}$ & 1981 & 1944 & & 2.9 \\
\hline & $3 / 2^{+}$ & 0 & 0 & & 1.4 \\
\hline \multirow[t]{3}{*}{${ }^{35} \mathrm{Si}$} & $7 / 2^{-}$ & 0 & 0 & 4.5 & 7.4 \\
\hline & $3 / 2^{-}$ & 910 & 909 & 2.8 & 3.7 \\
\hline & $3 / 2^{+}$ & 974 & 936 & & \\
\hline \multirow[t]{3}{*}{${ }^{33} \mathrm{~S}$} & $7 / 2^{-}$ & 2935 & 2942 & 4.2 & 5.8 \\
\hline & $3 / 2^{-}$ & 3221 & 3386 & 3.5 & 2.3 \\
\hline & $3 / 2^{+}$ & 0 & 0 & 3.5 & 2.6 \\
\hline \multirow[t]{3}{*}{${ }^{35} \mathrm{~S}$} & $7 / 2^{-}$ & 1991 & 2042 & 5.4 & 6.4 \\
\hline & $3 / 2^{-}$ & 2348 & 2409 & 2.1 & 2.7 \\
\hline & $3 / 2^{+}$ & 0 & 0 & 1.7 & 1.5 \\
\hline \multirow[t]{3}{*}{${ }^{37} \mathrm{~S}$} & $7 / 2^{-}$ & 0 & 0 & 5.5 & 7.3 \\
\hline & $3 / 2^{-}$ & 646 & 573 & 1.8 & 3.5 \\
\hline & $3 / 2^{+}$ & 1398 & 1303 & & \\
\hline \multirow[t]{3}{*}{${ }^{37} \mathrm{Ar}$} & $7 / 2^{-}$ & 1611 & 1543 & 6.1 & 6.3 \\
\hline & $3 / 2^{-}$ & 2491 & 2679 & 1.8 & 2.6 \\
\hline & $3 / 2^{+}$ & 0 & 0 & 2.2 & 1.5 \\
\hline \multirow[t]{3}{*}{${ }^{39} \mathrm{Ar}$} & $7 / 2^{-}$ & 0 & 0 & 5.0 & 6.7 \\
\hline & $3 / 2^{-}$ & 1267 & 1186 & 2.0 & 2.8 \\
\hline & $3 / 2^{+}$ & 1517 & 1457 & & \\
\hline
\end{tabular}

the $0 f_{7 / 2}$ and $1 p_{3 / 2}$ orbitals, the crossing between $0 f_{7 / 2}$ and $1 p_{3 / 2}$ happens between $Z=10$ and 12 . Together, these results show that the trend is robust, but the question of the relative position of the orbitals is more complex and nuanced than was expected earlier. 


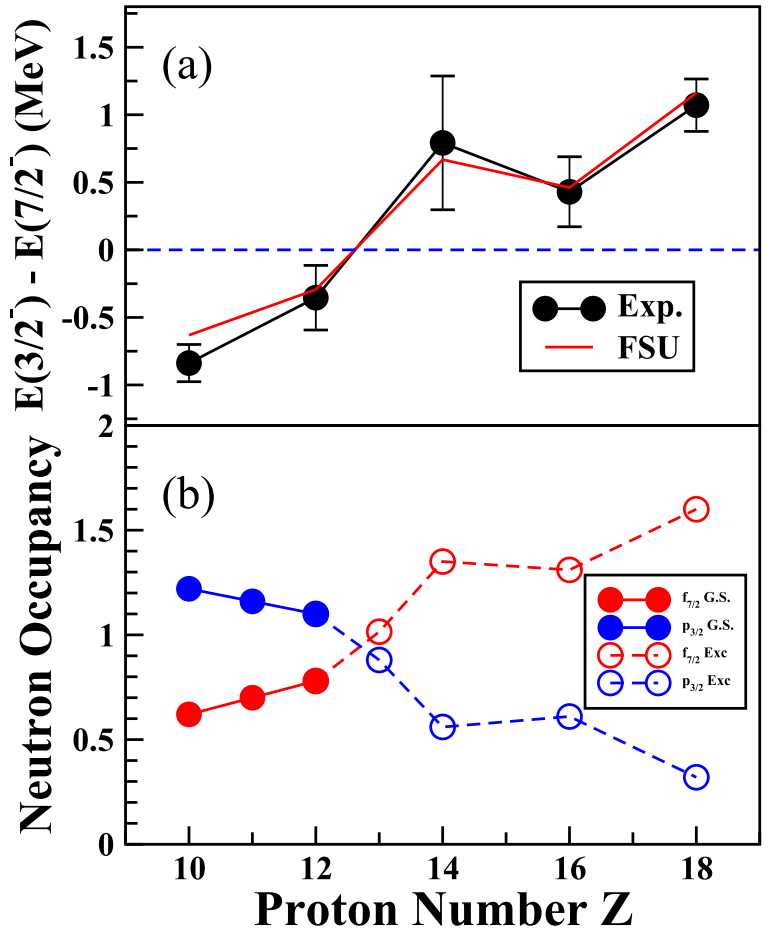

FIG. 3. (a) Average energy differences between the lowest $7 / 2^{-}$ and $3 / 2^{-}$experimental levels in Table I. The error bars give an indication of the range of values for different neutron numbers. Positive (negative) values of the ordinate correspond to the $3 / 2^{-}$state above (below) the $7 / 2^{-}$one. (b) Occupancies of the neutron $0 f_{7 / 2}$ and $1 p_{3 / 2}$ orbitals in neutron number $N=20$ nuclei as a function of proton number $Z$ for the lowest $2 \mathrm{p} 2 \mathrm{~h}$ states. The values are shown as filled circles for the cases where the lowest $2 \mathrm{p} 2 \mathrm{~h}$ state is the ground state (IoI) and as open circles where the lowest $2 \mathrm{p} 2 \mathrm{~h}$ state is excited above the ground state.

\section{EVOLUTION OF THE $N=20$ SHELL GAP AND THE ISLAND OF INVERSION}

One of the first indications that the pure $s d$ shell model could not represent low-lying states in all $s d$ nuclei came from the experimentally measured mass of ${ }^{31} \mathrm{Na}$ [14]. The experimental mass was about $1.6 \mathrm{MeV}$ lower than that predicted from the USD interaction [1]. This was further clarified by the USDA and USDB, showing that states in the highest $N-Z$ nuclei cannot be fitted. A consistent overprediction of 1 to 2 $\mathrm{MeV}$ of the ground-state energies of these nuclei can be seen in Fig. 9 of Ref. [2]. This region of nuclei is now known as the IoI and its origin has been discussed a lot. Most explanations center around the filled or almost-filled neutron $s d$ shell and $f p$ intruder configurations leading, counterintuitively, to lowering the energy of the two-particle, two -hole ( $2 \mathrm{p} 2 \mathrm{~h})$ state, with two nucleons being promoted from the $s d$ to $f p$ shell, below that of the "normal" $0 \mathrm{p} 0 \mathrm{~h}$. Such lowering is associated with increased correlation energy or higher deformation, lowering Nilsson orbitals. However, the effect fades away with filling of the proton $s d$ shell.

While a number of shell-model calculations in the past have reproduced many aspects of the IoI, as discussed in the Introduction, here we study what the FSU interaction predicts for the Iol nuclei. Concentrating on the IoI region, we consider

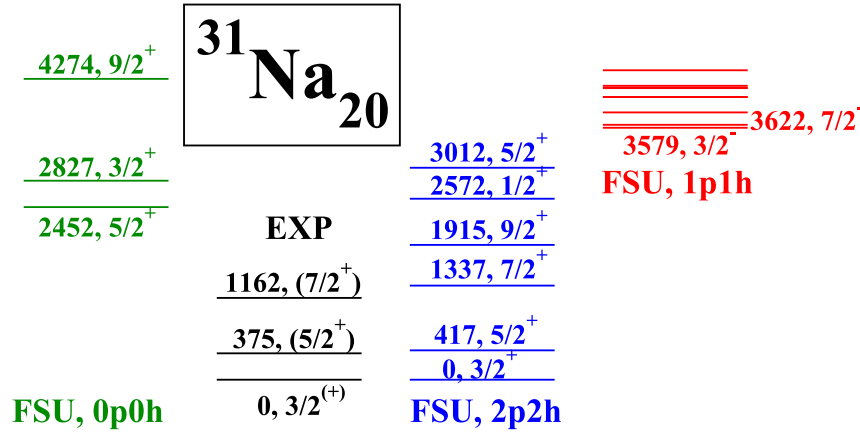

FIG. 4. The experimentally known levels of ${ }^{31} \mathrm{Na}$ compared to the lowest ones predicted using the FSU interaction for $0 \mathrm{p} 0 \mathrm{~h}, 1 \mathrm{p} 1 \mathrm{~h}$, and $2 \mathrm{p} 2 \mathrm{~h}$ configurations. The experimental levels agree well with the $2 \mathrm{p} 2 \mathrm{~h}$ results while the $0 \mathrm{p} 0 \mathrm{~h}$ states start almost $2.5 \mathrm{MeV}$ higher in excitation energy. Only the two lowest calculated $1 \mathrm{p} 1 \mathrm{~h}$ states are labeled because of the high-level density above this.

the states where two nucleons are promoted from $s d$ to $f p$, referring to them as $2 \mathrm{p} 2 \mathrm{~h}$ states. These states were not a part of the fit and, for this extrapolation to be meaningful, the additional $2 \mathrm{p} 2 \mathrm{~h}$ states cannot be allowed to directly mix and renormalize the previously fitted $0 \mathrm{p} 0 \mathrm{~h}$ states. Due to the valence space limitation, the full $2 \hbar \omega$ excitations from the $s d$ space cannot be considered. Moreover, our tests have shown that excitations from $0 s$ and $0 p$ are nearly irrelevant for the validity of this discussion, thus we did not include those states into our definition of $2 \mathrm{p} 2 \mathrm{~h}$ excitations. It also has been verified that the inevitable center-of-mass contamination in this truncation scheme is very low. We estimate that the errors from truncation and center-of-mass contamination amount to less then $200 \mathrm{keV}$ uncertainty in the energies, which is of the same order as the rms deviation in the fit.

We first discuss the case of ${ }^{31} \mathrm{Na}(N=20)$ [14]. As shown in Fig. 4, the total binding energies for the first four $2 \mathrm{p} 2 \mathrm{~h}$ states were found to be below that of the lowest $0 \mathrm{p} 0 \mathrm{~h}$ state. The first three $2 \mathrm{p} 2 \mathrm{~h}$ states agree well with what is so far known experimentally, whereas the lowest 0 p0h state $\left(5 / 2^{+}\right)$ appears much higher in energy and has a different spin from the experimentally observed ground state of ${ }^{31} \mathrm{Na}$.

While the experimental information is limited, it is clear that the FSU interaction has depicted the correct picture of ${ }^{31} \mathrm{Na}$ as one with the inverted configuration. As mentioned above, only the low $Z$ and $N \approx 20$ nuclei exhibit the IoI or inverted $2 \mathrm{p} 2 \mathrm{~h}-0 \mathrm{p} 0 \mathrm{~h}$ behavior. To explore the transition from IoI to "normal" behavior, Fig. 5 compares experimentally measured energies with our calculations for the lowest levels in a sequence of $N=20$ even- $A s d$ nuclei. For $Z=10$ and 12 , not only do the lowest states have $2 \mathrm{p} 2 \mathrm{~h}$ character, but the whole $0^{+}, 2^{+}, 4^{+} 2 \mathrm{p} 2 \mathrm{~h}$ sequence agrees well with experiment. In addition to starting much higher in energy, the spacing between $0 \mathrm{p} 0 \mathrm{~h}$ states differs significantly from experiment. The story changes for $Z=14{ }^{34} \mathrm{Si}$, where the $0 \mathrm{p} 0 \mathrm{~h} 0^{+}$state is substantially lower than the $2 \mathrm{p} 2 \mathrm{~h}$ one. The experimental second $0^{+}$and first $2^{+}$states are much closer to the $2 \mathrm{p} 2 \mathrm{~h}$ ones, while the second experimental $2^{+}$level corresponds well with the $0 \mathrm{p} 0 \mathrm{~h}$ one. This shows the shape coexistence, also discussed in Ref. [30]. For $Z=16$ and 18, both the first experimental $0^{+}$and $2^{+}$states correspond with 


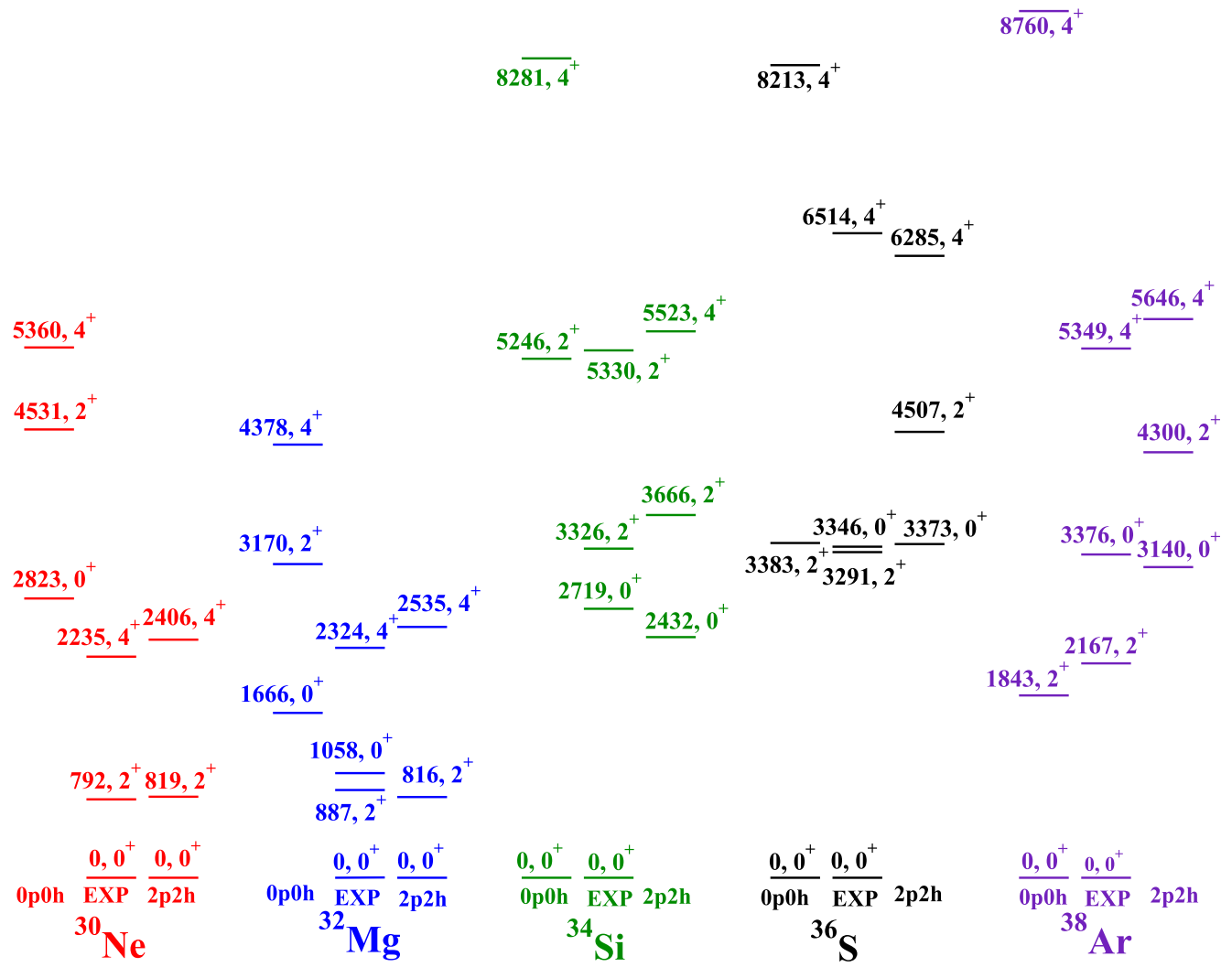

FIG. 5. The lowest experimental energy levels of $N=20 s d$-shell nuclei compared to those calculated using the FSU shell-model interaction for $0 \mathrm{p} 0 \mathrm{~h}$ and $2 \mathrm{p} 2 \mathrm{~h}$ configurations. The levels of the known IoI nuclei ${ }^{30} \mathrm{Ne}$ and ${ }^{32} \mathrm{Mg}$ agree well with the $2 \mathrm{p} 2 \mathrm{~h}$ results while the lowest states in the higher $\mathrm{Z}$ nuclei agree much better with the $0 \mathrm{p} 0 \mathrm{~h}$ results.

the $0 \mathrm{p} 0 \mathrm{~h}$ calculations. The second $0^{+}$states in both the nuclei were discussed to have $2 \mathrm{p} 2 \mathrm{~h}$ dominant configurations [31-33] and are in very good agreement with the FSU predictions. The $4^{+}$states of ${ }^{36} \mathrm{~S}$ and ${ }^{38} \mathrm{Ar}$ lie much closer to the calculated $2 \mathrm{p} 2 \mathrm{~h}$ ones. Note that the FSU cross-shell interaction describes the transition from inverted $0 \mathrm{p} 0 \mathrm{~h}-2 \mathrm{p} 2 \mathrm{~h}$ order to normal as a function of $Z$ despite not having been fitted to any of these states.

This emergence of the IoI does not involve any $f p$ orbitals dropping below the $s d$ shell, at least not for a spherical shape. The lowering in energy of the $2 \mathrm{p} 2 \mathrm{~h}$ configurations does not extend so much to $1 \mathrm{p} 1 \mathrm{~h}$ ones, as shown for ${ }^{31} \mathrm{Na}$ in Fig. 4. The lowest $1 \mathrm{p} 1 \mathrm{~h}$ state $\left(3579 \mathrm{keV}, 3 / 2^{-}\right)$lies over an $\mathrm{MeV}$ above the lowest $0 \mathrm{p} 0 \mathrm{~h}$ state. So, it is the promotion of a neutron pair to the $f p$ shell which favors the $2 \mathrm{p} 2 \mathrm{~h}$ configuration so much. The promotion of a neutron pair to the $f p$ orbital appears to lower its energy because of correlation energy in the shell model. Clearly, collective behaviors such as pairing and deformation and intricate interplay between them are central for the IoI phenomenon. Representing a mesoscopic phase transition, the picture is highly sensitive to the matrix elements of the effective Hamiltonian and, in particular, to the components describing short- and long-range limits of nucleon-nucleon in-medium interaction.

In a geometrical picture, IoI can be associated with increased prolate deformation due to the promotion of a pair into a downsloping Nilsson orbital whose excitation energy decreases rapidly with increasing deformation. An indication of this difference in deformation is shown in the lower panel of Fig. 6. For ${ }^{30} \mathrm{Ne}$ and ${ }^{32} \mathrm{Mg}$, the calculated B(E2) transition strengths from the lowest $2^{+}$to ground states (both of which have $2 \mathrm{p} 2 \mathrm{~h}$ configurations) are relatively large at over 400 $\mathrm{e}^{2} \mathrm{fm}^{4}$, consistent with relatively high deformation, and agree well with experiments. In contrast, the B(E2) strengths for ${ }^{36} \mathrm{~S}$ and ${ }^{38} \mathrm{Ar}$ are rather low, consistent with a near-spherical shape.

Figure 7 portrays the differences between experiment and theory of the binding energies around the IoI which are sensitive to pairing correlations. The calculated total binding energies are compared with the measured ground-state masses from the 2016 mass evaluation [34]. The Coulomb corrections to the total binding energies are included following procedures in Refs. $[1,2,15]$. The $N=210 \mathrm{p} 0 \mathrm{~h}(2 \mathrm{p} 2 \mathrm{~h})$ configurations have 1(3) nucleons in $f p$, and $N=22$ 2p2h actually have four $f p$ nucleons so the $f p$ matrix elements are tested along with the cross-shell ones. Looking at the $N=20$ isotonic chain, the agreement is quite good with an RMS deviation of 276 $\mathrm{keV}$, comparing the experimental binding energies with the $2 \mathrm{p} 2 \mathrm{~h}$ results below $Z=13$ and with $0 \mathrm{p} 0 \mathrm{~h}$ for higher $Z$. For $10 \leqslant Z \leqslant 12$ and $19 \leqslant N \leqslant 21$, the $2 \mathrm{p} 2 \mathrm{~h}$ inverted configuration is lower in energy and agrees better with experiments. Outside this range, the $0 \mathrm{p} 0 \mathrm{~h}$ configuration is lower, which again agrees with experiments. For $N=22$, it appears that promoting a second neutron pair to $f p$ is not energetically favorable.

A similar approach of calculating the $2 \mathrm{p} 2 \mathrm{~h}$ states was taken in Ref. [15] using the WBMB interaction. As mentioned earlier, the WBMB interaction was developed for the 


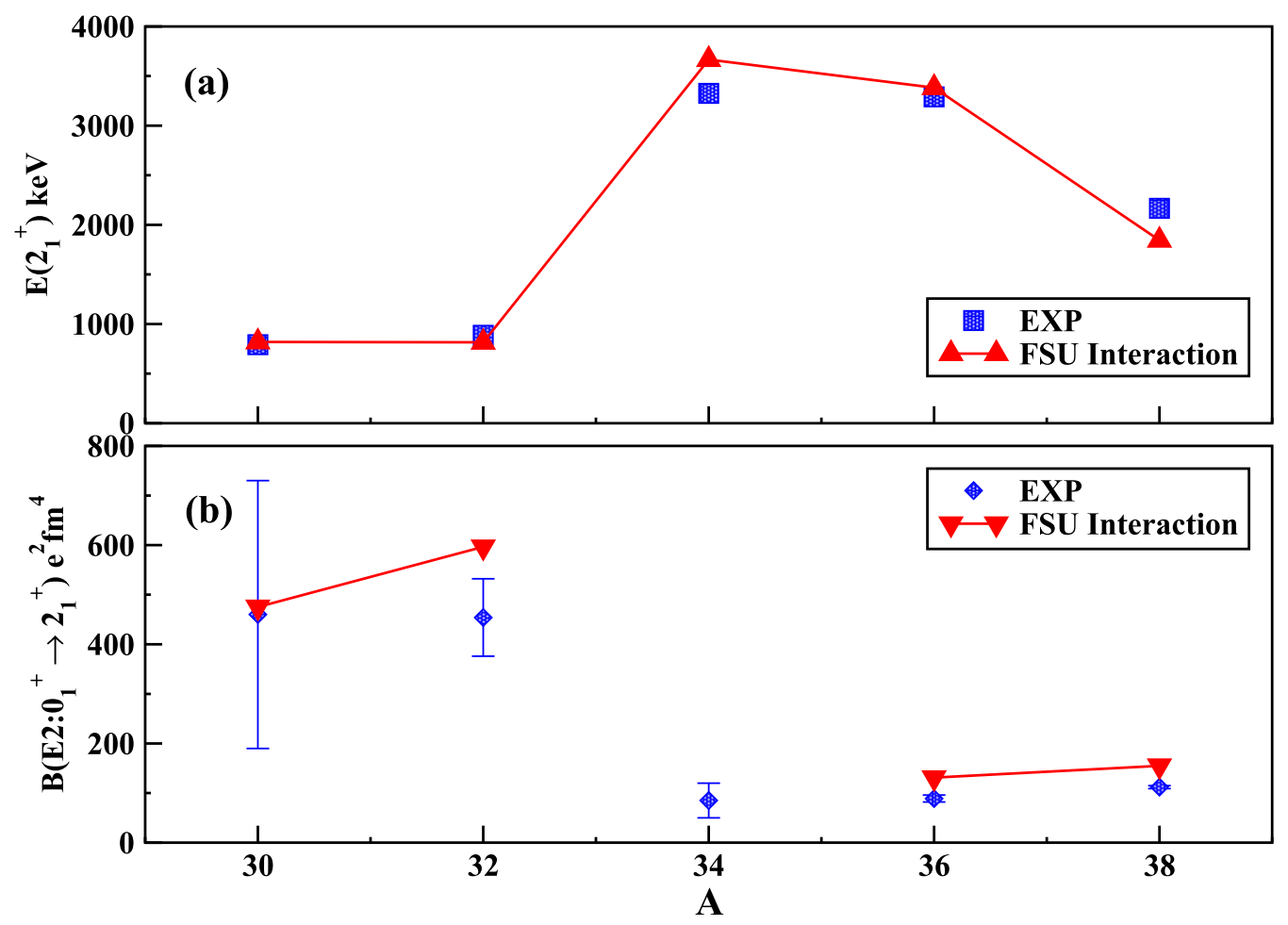

FIG. 6. Experimental $\mathrm{E}\left(2_{1}^{+}\right)$and $\mathrm{B}\left(\mathrm{E} 2: 0_{1}^{+} \rightarrow 2_{1}^{+}\right)$values for the $N=20$ isotones are compared to those calculated by using the FSU interaction. The $\mathrm{B}\left(\mathrm{E} 2: 0_{1}^{+} \rightarrow 2_{1}^{+}\right)$value of ${ }^{34} \mathrm{Si}$ has not been calculated because of the different configurations associated with the $0_{1}^{+}$and $2_{1}^{+}$ states.

mass region near ${ }^{40} \mathrm{Ca}$ by fitting the $1 \mathrm{p} 1 \mathrm{~h}$ states within the $s d f p$ model space. We have compared the differences in the $0 \mathrm{p} 0 \mathrm{~h}$ and $2 \mathrm{p} 2 \mathrm{~h}$ ground states calculated by using the WBMB and the FSU interactions for $N=20$ isotones in Table II. The predictions with the WBMB interaction were taken from Ref. [15]. From Table II, we see that both interactions predict ${ }^{30} \mathrm{Ne},{ }^{31} \mathrm{Na}$, and ${ }^{32} \mathrm{Mg}$ having their $2 \mathrm{p} 2 \mathrm{~h}$ ground state more tightly bound than that calculated for the $0 \mathrm{p} 0 \mathrm{~h}$ configurations,

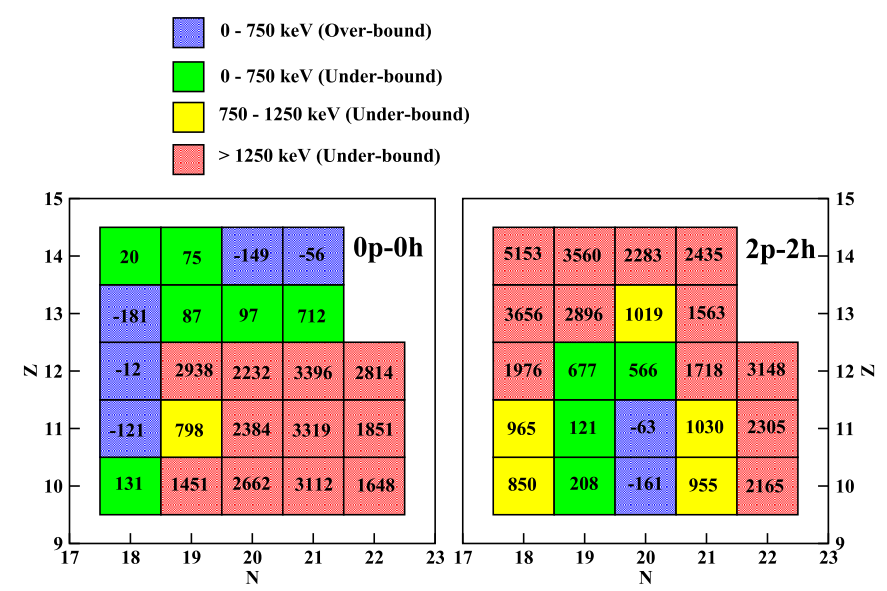

FIG. 7. The number displayed inside a box corresponding to an isotope is the difference in binding energy between experiments and shell-model predictions using the FSU interaction with 0 or 2 particle-hole configurations. We call the states overbound where the calculated states are more tightly bound than that of the experimental ones and underbound when it is otherwise. meaning that these nuclei are the members of the IoI. The FSU interaction predicts ${ }^{29} \mathrm{~F}$ also as an IoI nucleus, which was suggested recently by Ref. [35]. The difference between the

TABLE II. The ground-state energies with $2 \mathrm{p} 2 \mathrm{~h}$ configurations are calculated with respect to those with the $0 \mathrm{p} 0 \mathrm{~h}$ configurations using the WBMB [15] and FSU interactions. The symbols W, F, and $\mathrm{T}$ in the WBMB calculations stand for weak coupling, full WBMB space, and the truncated space, respectively.

\begin{tabular}{|c|c|c|}
\hline Nucleus & $\mathrm{WBMB}^{\mathrm{a}}$ & FSU \\
\hline${ }^{28} \mathrm{O}$ & $\begin{array}{l}\text { 3038: } \mathrm{W} \\
\text { 2956: } \mathrm{F}\end{array}$ & -755 \\
\hline${ }^{29} \mathrm{~F}$ & $\begin{array}{l}\text { 1286: W } \\
\text { 1338: F }\end{array}$ & -2201 \\
\hline${ }^{30} \mathrm{Ne}$ & $\begin{array}{l}-698: \mathrm{W} \\
-788: \mathrm{F}\end{array}$ & -2823 \\
\hline${ }^{31} \mathrm{Na}$ & $\begin{array}{l}-502: \mathrm{W} \\
-764: \mathrm{T}\end{array}$ & -2452 \\
\hline${ }^{32} \mathrm{Mg}$ & $\begin{array}{l}-926: \mathrm{W} \\
-966: \mathrm{T}\end{array}$ & -1666 \\
\hline${ }^{33} \mathrm{Al}$ & 854: W & 922 \\
\hline${ }^{34} \mathrm{Si}$ & $\begin{array}{l}\text { 1816: W } \\
\text { 1554: T }\end{array}$ & 2432 \\
\hline${ }^{35} \mathrm{P}$ & 2698: W & 3264 \\
\hline${ }^{36} \mathrm{~S}$ & $\begin{array}{l}\text { 3146: W } \\
\text { 3009: T }\end{array}$ & 3373 \\
\hline${ }^{37} \mathrm{Cl}$ & $\begin{array}{l}\text { 3195: W } \\
\text { 3091: T }\end{array}$ & 3538 \\
\hline${ }^{38} \mathrm{Ar}$ & 2701: F & 3140 \\
\hline
\end{tabular}

${ }^{a}$ From Ref. [15]. 

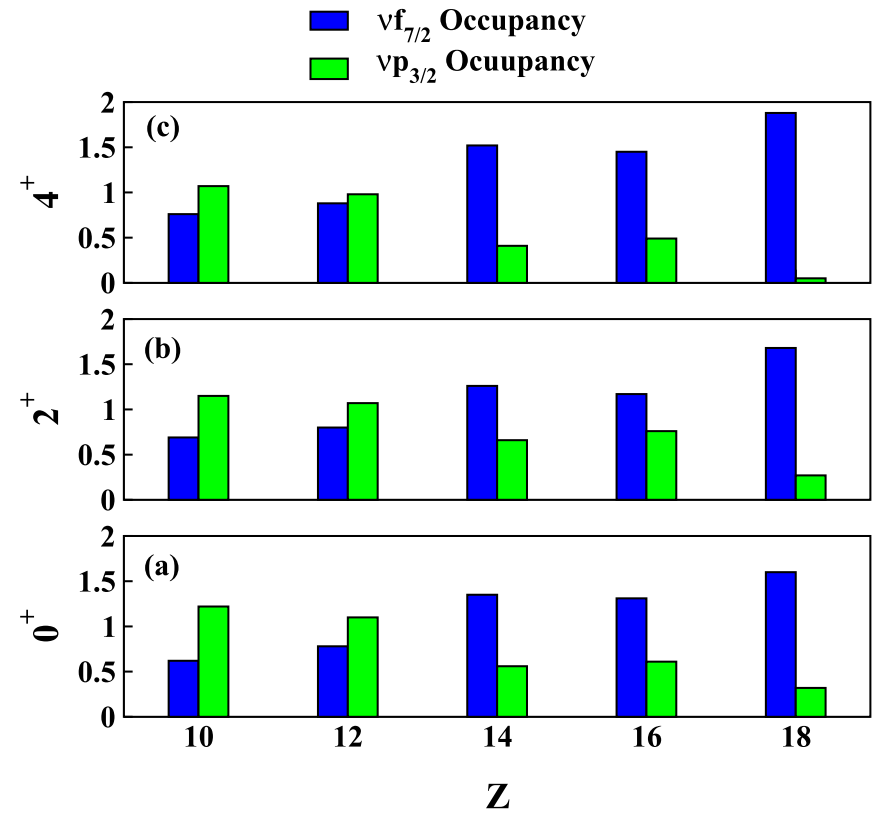

FIG. 8. 2p2h occupancies of the $v 0 f_{7 / 2}$ and $v 1 p_{3 / 2}$ orbitals for the first $0^{+}, 2^{+}$, and $4^{+}$calculated states using the FSU interaction for nuclei with $N=20$ and $Z$ between 10 and 18 .

first two $0^{+}$states in ${ }^{34} \mathrm{Si}$ is known experimentally as 2719 $\mathrm{keV}$ [30]. The FSU interaction predicts it better as $2432 \mathrm{keV}$. The experimentally observed $0_{2}^{+}$states in ${ }^{36} \mathrm{~S}$ and ${ }^{38} \mathrm{Ar}$ are at 3346 and $3376 \mathrm{keV}$, respectively, which are presumably $2 \mathrm{p} 2 \mathrm{~h}$ in nature. The FSU interaction predicts them at 3373 and 3140 $\mathrm{keV}$, respectively. In ${ }^{37} \mathrm{Cl}$, the first $2 \mathrm{p} 2 \mathrm{~h}$ state was identified at $3708 \mathrm{keV}$ energy [36], whereas the FSU prediction is 3538 $\mathrm{keV}$. The better predictability of the FSU interaction comes from a more extensive fit for a wide range of cross-shell data as well as the use of a better Hamiltonian for the $f p$ shell, we believe.

Since the IoI involves excitations into the $f p$ shell, the question arises how the inversion of the $0 f_{7 / 2}$ and $1 p_{3 / 2}$ SPEs at low $Z$, discussed above, affects our understanding of the IoI. The answer, within the context of the FSU interaction, is shown in Fig. 8. This figure shows some of the $f p$ shell occupancies calculated for the lowest $2 \mathrm{p} 2 \mathrm{~h}$ states in Fig. 5. Occupancy here is defined as the average number of nucleons in a given orbital. There is almost no proton $f p$ occupancy calculated for these nuclei and there is a relatively constant $v 1 p_{1 / 2}$ occupancy of about 0.1 neutron. For $Z=10{ }^{30} \mathrm{Ne}$, which is the most strongly inverted, the $v 1 p_{3 / 2}$ occupancy is about twice that of $\nu 0 f_{7 / 2}$. With increasing $Z$, the ratio of $\nu 1 p_{3 / 2}$ to $\nu 0 f_{7 / 2}$ decreases steadily from about 2 to about 0.2 across this region. Of course, the energies of the $2 \mathrm{p} 2 \mathrm{~h}$ configurations rise above that of the $0 \mathrm{p} 0 \mathrm{~h}$ ones around $Z=14$.

We note that, considering that the degeneracy of the $f_{7 / 2}$ is twice that of $p_{3 / 2}$, at the level crossing or in the limit of strong pairing the ratio of occupancies of $\nu 1 p_{3 / 2}$ to $\nu 0 f_{7 / 2}$ should be about 0.5. This indeed happens at around $Z=14$; however, significant deviation from 0.5 suggests that pairing or at least pair transfer between $f_{7 / 2}$ and $p_{3 / 2}$ is weak. Pair transfer and pair vibration, collective pairing condensation, interplay of paring and deformation in the IoI region, as well as the con- nection of these collective effects with the underlying matrix elements of the FSU Hamiltonian all require more study and remain a challenge for the future. The occupancy trend is perhaps illustrated more clearly in Fig. 3(b), which shows the $v 1 p_{3 / 2}$ and $v 0 f_{7 / 2}$ occupancies of the lowest $2 \mathrm{p} 2 \mathrm{~h}$ states in the $N=20$ nuclei as a function of proton number $Z$. Note that for ${ }^{34} \mathrm{Si}$, the $2 \mathrm{p} 2 \mathrm{~h} 0^{+}$state lies $2432 \mathrm{keV}$ above the $0 \mathrm{p} 0 \mathrm{~h}$ ground state but the $2 \mathrm{p} 2 \mathrm{~h} 2^{+}$level lies close in energy with the lowest experimental $2^{+}$state. Together these calculations imply that the $v 1 p_{3 / 2}$ orbital plays a larger role in the IoI phenomenon than does the $\nu 0 f_{7 / 2}$ one.

\section{FULLY ALIGNED STATES}

In describing the states used in the fit of the FSU interaction, we included only $0 \mathrm{p} 0 \mathrm{~h}(1 \mathrm{p} 1 \mathrm{~h})$ configurations for natural (unnatural) parity sectors. In particular, no $2 \mathrm{p} 2 \mathrm{~h}$ configurations were used to adjust the interaction parameters. After the fitting, two early tests were performed to explore the predictive properties of the FSU interaction for $2 \mathrm{p} 2 \mathrm{~h}$ configurations. One was the calculation of the lowest $2 \mathrm{p} 2 \mathrm{~h} 7^{+}$states in ${ }^{34} \mathrm{Cl}$ and ${ }^{36} \mathrm{Cl}$ [17]. These agreed within $200 \mathrm{keV}$ with the experimental states. The other test was performed on ${ }^{38} \mathrm{Ar}$ [37], since experimental states up to $8^{+}$and $\left(10^{+}\right)$are known. Calculations using the USD family of interactions agree within $200 \mathrm{keV}$ with the excitation energy of the lowest $2^{+}$state of ${ }^{38} \mathrm{Ar}$ but overpredict the lowest experimental $4^{+}$level by over $3 \mathrm{MeV}$. With only two holes in the $s d$ shell, the maximum spin from coupling two $0 d_{3 / 2}$ protons is $2 \hbar$. The very high $4^{+}$energy represents the cost of promoting a $0 d_{5 / 2}$ proton to $0 d_{3 / 2}$, but nature finds another less energetic way of achieving $4^{+}$. This must be by promoting an $s d$ nucleon pair to the $f p$ shell. A 2p2h calculation with the FSU interaction predicts the lowest $4^{+}$level only $300 \mathrm{keV}$ above the experimental one, and it predicts the $6+$ state $200 \mathrm{keV}$ below experiment, while the predicted $8^{+}$state is $100 \mathrm{keV}$ above experiment as shown in Ref. [37].

With this success we have searched for other states with confirmed $2 \mathrm{p} 2 \mathrm{~h}$ structure to compare with theory. One such group of excited states across the $s d$ shell is often called the fully aligned states. One subgroup of fully aligned states is the lowest $J^{\pi}=7^{+}$states. These states have been suggested to have both odd nucleons in the highest spin orbital around$0 f_{7 / 2}$ - and with their spins fully aligned, which, from the Pauli principle, is only possible for nonidentical nucleons. For these calculations, it is critical that the FSU interaction treats protons and neutrons on an equivalent basis. These fully aligned $\pi f_{7 / 2} \otimes v f_{7 / 2}$ are yrast states and strongly populated in high-spin $\gamma$-decay sequences. Stronger evidence of their unique nature comes from $(\alpha, d)$ reactions [38-44] where they are the most strongly populated states with an orbital angular momentum transfer of $\ell=6$. In most cases, such states involve two nucleons beyond those in the dominant ground-state configuration outside the $s d$ shell. The energies of these $7^{+}$ states (including those in ${ }^{34} \mathrm{Cl}$ and ${ }^{36} \mathrm{Cl}$ mentioned above) are graphed in Fig. 9 along with calculated results using the FSU interaction. The agreement is excellent both in value and in the trend which extends from $10 \mathrm{MeV}$ for the lightest nuclei down to $2 \mathrm{MeV}$ for the heaviest and from $2 \mathrm{p} 2 \mathrm{~h}$ to $1 \mathrm{p} 1 \mathrm{~h}$ excitations relative to the ground state. The calculations also indirectly 


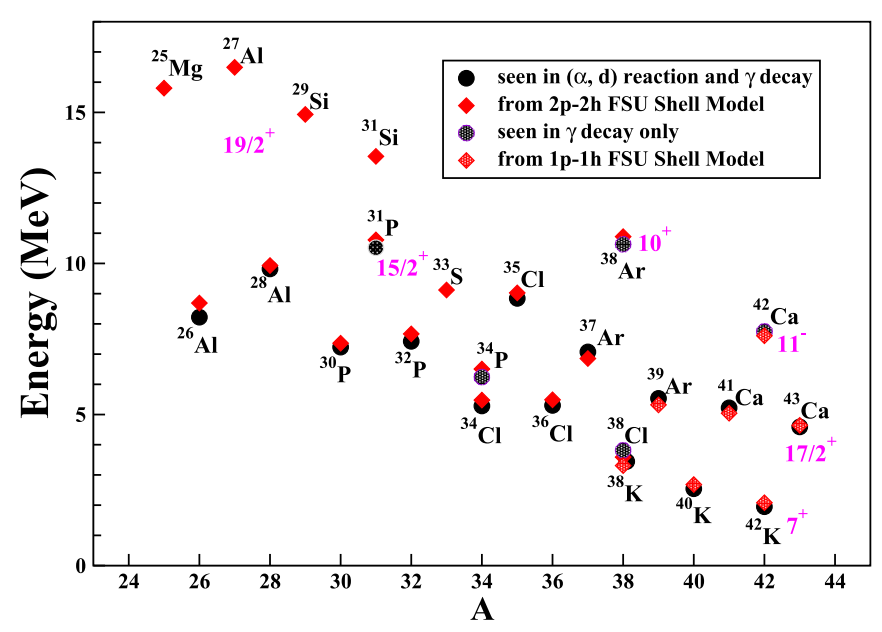

FIG. 9. Comparisons of the energies of fully aligned states in $s d$-shell nuclei with those predicted employing the FSU interaction. Many of the experimental points are confirmed by both selective population in $(\alpha, d)$ reactions and in high-spin $\gamma$ decay sequences and are displayed with solid black circles, while dotted black circles are used to represent states observed by only one of the two signatures. The structure of many of these aligned states involve the promotion of two (extra) nucleons to the $0 f_{7 / 2}$ orbital and are shown with solid red diamonds. Those with at least one nucleon in the $0 f_{7 / 2}$ orbital may require only one more promotion (1p1h excitation) and are shown with dotted red diamond symbols.

confirm the spin alignment with approximately equal proton and neutron occupancies in the $0 f_{7 / 2}$ orbitals, even though most $2 \mathrm{p} 2 \mathrm{~h}$ states in these neutron-rich nuclei as discussed in the IoI section involve predominantly two neutron configurations.

Fully aligned states are also known for some odd- $A$ nuclei where an $s d$ nucleon is also aligned in spin with the aligned $0 f_{7 / 2}$ nucleons. Five such cases in Fig. 9 are known experimentally as the strongest states populated in $(\alpha, d)$ reactions. They have an unpaired nucleon in the $0 d_{3 / 2}$ orbital which contributes an extra spin of $3 / 2 \hbar$. Again the $2 \mathrm{p} 2 \mathrm{~h}$ and $1 \mathrm{p} 1 \mathrm{~h}$ calculations with the FSU interaction agree well. In lighter odd-A nuclei, the aligned $s d$ nucleon could be in the $1 s_{1 / 2}$ or $0 d_{5 / 2}$ orbitals, leading to total spins of $15 / 2$ or $19 / 2$ and higher excitation energies. Their calculated energies are also shown in Fig. 9, but none have been seen in $(\alpha, d)$ reactions. A $\left(11 / 2^{+}, 15 / 2^{+}\right)$state which decays only to the lowest $13 / 2^{+}$ state and is very likely the $15 / 2^{+}$fully aligned state has been reported [24] in ${ }^{31} \mathrm{P}$ and, as shown in the figure, would agree well with the predictions.

The last category of aligned states in the $s d$ shell consists of those in even-even nuclei. Their excitations involve the breaking of a proton and a neutron pair and promotion of one of each nucleon to the $0 f_{7 / 2}$ orbital. For example, all four unpaired nucleons couple to maximum spin of $10^{+}$if both unpaired $s d$ nucleons are in the $0 d_{3 / 2}$ orbital. No $(\alpha, d)$ reactions to the fully aligned state in even-even nuclei are known because of the absence of stable odd- $Z$, odd- $N$ targets in the $s d$ shell. However, the lowest experimentally known $10^{+}$state in ${ }^{38} \mathrm{Ar}$ observed by other reactions does compare well with a $2 \mathrm{p} 2 \mathrm{~h}$ calculation using the FSU interaction, as shown in
Fig. 9. In the case of ${ }^{42} \mathrm{Ca}$, the analogous state would involve breaking a $\pi d_{3 / 2}$ pair, promoting one proton to $0 f_{7 / 2}$, breaking the $v f_{7 / 2}$ pair, and coupling them to maximum spin for a total of $11^{-}$. This state has been seen in $\gamma$ decay following fusion evaporation and its energy agrees well with the FSU calculation. We hope that future experiments in the Facility of Rare Isotope Beams (FRIB) age will be able to test these predictions. This study of aligned states targets cross-shell matrix elements of high angular momentum channels that describe long-range effective in-medium nucleon-nucleon interactions and play a key role in determining nuclear shape and deformation.

\section{SUMMARY}

In this paper, we presented an effective nuclear interaction Hamiltonian for shell-model calculations, called FSU interaction. The interaction targets a broad range of nuclei from $p$ to $f p$ shells with a particular emphasis on exotic nuclei with extreme-proton to-neutron ratios and on states that involve cross-shell excitations. The interaction was fitted using binding energies and $1 \hbar \omega$ states that probe cross-shell matrix elements in nuclei from ${ }^{13} \mathrm{C}$ through ${ }^{51} \mathrm{Ti}$ and ${ }^{49} \mathrm{~V}$. Additional details of the fit can be found in Refs. [17,23]. This report provides a comprehensive study of nuclei in the region of the IoI, namely, those nuclei between $s d$ and $f p$ shells whose low-lying structure is dominated by cross shell excitations.

We use the obtained FSU interaction to infer information about the mean field and evolution of the ESPEs. The ESPEs of the $0 f_{7 / 2}$ and $1 p_{3 / 2}$ show the expected normal ordering, where $0 f_{7 / 2}$ is below $1 p_{3 / 2}$ for $Z>12$ and a consistent trend of a decreasing separation with decreasing $Z$ until the energy order reverses around $Z=10$ to 12 . It is remarkable that the inversion happens near zero energy associated with the decay threshold. The interaction with the continuum is not explicitly included but may be captured as a part of the fit. While there have been many indications of inverted shell ordering in the past, these results present a more systematic picture from a model very firmly rooted in data. Perhaps somewhat surprisingly, over the range explored here, the inversion appears to depend more on the proton number than on the neutron excess. The lowest $3 / 2^{+}, 7 / 2^{-}$, and $3 / 2^{-}$experimental states are surveyed for a complementary view of shell evolution. These energies are compared with predictions of the FSU interaction in excitation energies and SFs. They present a similar picture of the $0 f_{7 / 2}-1 p_{3 / 2}$ shell evolution as a function of proton number.

The success of the FSU interaction in reproducing the negative parity states of the $s d$-shell nuclei with the $1 \hbar \omega$ configuration suggests that improved, over those in Ref. [45], calculations of the rp-process rates can be performed in the future.

In this paper, the FSU interaction was taken a step forward and applied to configurations involving promotion of two nucleons from $s d$ to $f p(2 \mathrm{p} 2 \mathrm{~h})$ in the region of IoI. In this region, the nuclei are more tightly bound than predicted within the pure $s d$ model space $(0 \mathrm{p} 0 \mathrm{~h})$. The $2 \mathrm{p} 2 \mathrm{~h}$ configurations have lower binding energies and agree well with the measured ground-state masses in the range $10 \leqslant Z \leqslant 12$ and $19 \leqslant N \leqslant$ 
21 , while the 0 p0h configurations are lower in energy and agree better with the measured masses elsewhere. The lowest $2^{+}$states agree well with the $2 \mathrm{p} 2 \mathrm{~h}$ calculations in the region $Z=14$ and with 0 p0h for $Z=16-18$. The results of the FSU interaction which were not fitted to these states reproduce well both the IoI and the transition to normal behavior. ${ }^{34} \mathrm{Si}$ with $Z=14$ emerges as transitional with a $0 \mathrm{p} 0 \mathrm{~h}$ ground state and a $2 \mathrm{p} 2 \mathrm{~h}$ lowest $2^{+}$state. It would be interesting to locate the experimental $4_{1}^{+}$state which is predicted as $2 \mathrm{p} 2 \mathrm{~h}$ at 5523 $\mathrm{keV}$. Another implication of the FSU shell-model calculations is that $v 1 p_{3 / 2}$ pairs dominate over $v 0 f_{7 / 2}$ ones in the IoI, but $\nu 0 f_{7 / 2}$ pairs dominate the lowest $2 \mathrm{p} 2 \mathrm{~h}$ states beyond the IoI. This is an indication of a relative weakness of pairing that would act to equilibrate occupancy. Interestingly, the IoI coincides relatively well with the region where the $v 1 p_{3 / 2}$ orbital falls below the $v 0 f_{7 / 2}$ one.

Another success of the FSU interaction has been the calculation of the energies and occupancies of the fully aligned states, first identified in the early 1960s in $(\alpha, d)$ reactions and frequently observed in high-spin $\gamma$-decay cascades (most involve $2 \mathrm{p} 2 \mathrm{~h}$ excitations relative to the ground state). Their energies are reproduced very well across the mass range, and their occupancies prove the excitation of both protons and neutrons, even though pure neutron excitations are more common in other states. This is an important result that establishes values for the specific cross-shell high angular momentum matrix elements that are responsible for long-range effective nucleon-nucleon interaction and are particularly challenging to obtain from fundamental principles.

This paper brings forward an interesting comparison between traditional shell model interactions with those arising from first-principles methods. While the former are obtained from simply fitting SPEs and TBMEs to experimental data, the latter require renormalizations, many-body forces, and explicit inclusion of the reaction continuum to achieve agreement with experiment. This dichotomy presents a modern challenge to nuclear theory and deserves full investigation.

The capability of the FSU interaction to explain the exotic phenomena of the nuclei carries the prospect that the interaction will be successful for more exotic nuclei or states. It is hoped that the interaction will prove valuable in the coming FRIB age.

\section{ACKNOWLEDGMENTS}

This work was supported by U.S. National Science Foundation under Grant No. PHY-1712953 (FSU), U.S. Department of Energy, office of Science, under Award No. DE-SC-0009883 (FSU). Part of the paper was prepared at LLNL under Contract No. DE-AC52-07NA27344.
[1] B. Wildenthal, Empirical strengths of spin operators in nuclei, Prog. Part. Nucl. Phys. 11, 5 (1984).

[2] B. A. Brown and W. A. Richter, New USD Hamiltonians for the sd shell, Phys. Rev. C 74, 034315 (2006).

[3] T. Motobayashi, Y. Ikeda, K. Ieki, M. Inoue, N. Iwasa, T. Kikuchi, M. Kurokawa, S. Moriya, S. Ogawa, H. Murakami, S. Shimoura, Y. Yanagisawa, T. Nakamura, Y. Watanabe, M. Ishihara, T. Teranishi, H. Okuno, and R. Casten, Large deformation of the very neutron-rich nucleus ${ }^{32} \mathrm{Mg}$ from intermediate-energy Coulomb excitation, Phys. Lett. B 346, 9 (1995).

[4] G. Huber, F. Touchard, S. Büttgenbach, C. Thibault, R. Klapisch, H. T. Duong, S. Liberman, J. Pinard, J. L. Vialle, P. Juncar, and P. Jacquinot, Spins, magnetic moments, and isotope shifts of ${ }^{21-31} \mathrm{Na}$ by high resolution laser spectroscopy of the atomic $D_{1}$ line, Phys. Rev. C 18, 2342 (1978).

[5] C. Détraz, D. Guillemaud, G. Huber, R. Klapisch, M. Langevin, F. Naulin, C. Thibault, L. C. Carraz, and F. Touchard, Beta decay of ${ }^{27-32} \mathrm{Na}$ and their descendants, Phys. Rev. C 19, 164 (1979).

[6] D. Guillemaud-Mueller, C. Detraz, M. Langevin, F. Naulin, M. de Saint-Simon, C. Thibault, F. Touchard, and M. Epherre, $\beta$ decay schemes of very neutron-rich sodium isotopes and their descendants, Nucl. Phys. A 426, 37 (1984).

[7] Y. Yanagisawa, M. Notani, H. Sakurai, M. Kunibu, H. Akiyoshi, N. Aoi, H. Baba, K. Demichi, N. Fukuda, H. Hasegawa, Y. Higurashi, M. Ishihara, N. Iwasa, H. Iwasaki, T. Gomi, S. Kanno, M. Kurokawa, Y. Matsuyama, S. Michimasa, T. Minemura, T. Mizoi, T. Nakamura, A. Saito, M. Serata, S. Shimoura, T. Sugimoto, E. Takeshita, S. Takeuchi, K. Ue, K. Yamada, K. Yoneda, and T. Motobayashi, The first excited state of ${ }^{30} \mathrm{Ne}$ studied by proton inelastic scattering in reversed kinematics, Phys. Lett. B 566, 84 (2003).

[8] V. Tripathi, S. L. Tabor, P. Bender, C. R. Hoffman, S. Lee, K. Pepper, M. Perry, P. F. Mantica, J. M. Cook, J. Pereira, J. S. Pinter, J. B. Stoker, D. Weisshaar, Y. Utsuno, and T. Otsuka, Excited intruder states in ${ }^{32} \mathrm{Mg}$, Phys. Rev. C 77, 034310 (2008).

[9] E. Caurier, F. Nowacki, A. Poves, and J. Retamosa, Shell model study of the neutron rich isotopes from oxygen to silicon, Phys. Rev. C 58, 2033 (1998).

[10] F. Nowacki and A. Poves, New effective interaction for $\mathbf{0} \hbar \omega$ shell-model calculations in the sd - pf valence space, Phys. Rev. C 79, 014310 (2009).

[11] M. Bouhelal, F. Haas, E. Caurier, F. Nowacki, and A. Bouldjedri, A PSDPF interaction to describe the $1 \hbar \omega$ intruder states in sd shell nuclei, Nucl. Phys. A 864, 113 (2011).

[12] Y. Utsuno, T. Otsuka, T. Mizusaki, and M. Honma, Varying shell gap and deformation in $\mathrm{N} \sim 20$ unstable nuclei studied by the Monte Carlo shell model, Phys. Rev. C 60, 054315 (1999).

[13] E. Caurier, F. Nowacki, and A. Poves, Merging of the islands of inversion at $\mathrm{N}=20$ and $\mathrm{N}=28$, Phys. Rev. C 90, 014302 (2014).

[14] C. Thibault, R. Klapisch, C. Rigaud, A. M. Poskanzer, R. Prieels, L. Lessard, and W. Reisdorf, Direct measurement of the masses of ${ }^{11} \mathrm{Li}$ and ${ }^{26-32} \mathrm{Na}$ with an on-line mass spectrometer, Phys. Rev. C 12, 644 (1975).

[15] E. K. Warburton, J. A. Becker, and B. A. Brown, Mass systematics for $\mathrm{A}=29-44$ nuclei: The deformed $\mathrm{A} \sim 32$ region, Phys. Rev. C 41, 1147 (1990).

[16] N. Tsunoda, T. Otsuka, N. Shimizu, M. Hjorth-Jensen, K. Takayanagi, and T. Suzuki, Exotic neutron-rich medium-mass 
nuclei with realistic nuclear forces, Phys. Rev. C 95, 021304(R) (2017).

[17] R. S. Lubna, K. Kravvaris, S. L. Tabor, V. Tripathi, A. Volya, E. Rubino, J. M. Allmond, B. Abromeit, L. T. Baby, and T. C. Hensley, Structure of ${ }^{38} \mathrm{Cl}$ and the quest for a comprehensive shell model interaction, Phys. Rev. C 100, 034308 (2019).

[18] E. K. Warburton and B. A. Brown, Effective interactions for the 0p1s0d nuclear shell-model space, Phys. Rev. C 46, 923 (1992).

[19] P. C. Bender, C. R. Hoffman, M. Wiedeking, J. M. Allmond, L. A. Bernstein, J. T. Burke, D. L. Bleuel, R. M. Clark, P. Fallon, B. L. Goldblum, T. A. Hinners, H. B. Jeppesen, S. Lee, I.-Y. Lee, S. R. Lesher, A. O. Macchiavelli, M. A. McMahan, D. Morris, M. Perry, L. Phair, N. D. Scielzo, S. L. Tabor, V. Tripathi, and A. Volya, Approaching the "island of inversion": ${ }^{34}$ P, Phys. Rev. C 80, 014302 (2009).

[20] P.-L. Tai, S. L. Tabor, R. S. Lubna, K. Kravvaris, P. C. Bender, V. Tripathi, A. Volya, M. P. Carpenter, R. V. F. Janssens, T. Lauritsen, E. A. McCutchan, S. Zhu, R. M. Clark, P. Fallon, S. Paschalis, M. Petri, A. O. Macchiavelli, W. Reviol, and D. G. Sarantites, Cross-shell excitations in ${ }^{31} \mathbf{S i}$, Phys. Rev. C 96, 014323 (2017).

[21] S. M. Brown, W. N. Catford, J. S. Thomas, B. FernándezDomínguez, N. A. Orr, M. Labiche, M. Rejmund, N. L. Achouri, H. Al Falou, N. I. Ashwood, D. Beaumel, Y. Blumenfeld, B. A. Brown, R. Chapman, M. Chartier, N. Curtis, G. de France, N. de Sereville, F. Delaunay, A. Drouart, C. Force, S. Franchoo, J. Guillot, P. Haigh, F. Hammache, V. Lapoux, R. C. Lemmon, A. Leprince, F. Maréchal, X. Mougeot, B. Mouginot, L. Nalpas, A. Navin, N. P. Patterson, B. Pietras, E. C. Pollacco, A. Ramus, J. A. Scarpaci, I. Stefan, and G. L. Wilson, Low-lying neutron fp-shell intruder states in ${ }^{27} \mathrm{Ne}$, Phys. Rev. C 85, 011302(R) (2012).

[22] M. Honma, T. Otsuka, B. A. Brown, and T. Mizusaki, Shellmodel description of neutron-rich pf-shell nuclei with a new effective interaction GXPF1, Euro. Phys. J. A 25, 499 (2005).

[23] R. S. Lubna, Ph.D. thesis, Florida State University, 2019.

[24] http://www.nndc.bnl.gov/ensdf/.

[25] A. Volya, Continuum Shell Model code, https://www.volya. net/.

[26] N. A. Smirnova, K. Heyde, B. Bally, F. Nowacki, and K. Sieja, Nuclear shell evolution and in-medium $n n$ interaction, Phys. Rev. C 86, 034314 (2012).

[27] I. Talmi and I. Unna, Order of Levels in the Shell Model and Spin of Be ${ }^{11}$, Phys. Rev. Lett. 4, 469 (1960).

[28] C. R. Hoffman, B. P. Kay, and J. P. Schiffer, Neutron $s$ states in loosely bound nuclei, Phys. Rev. C 89, 061305(R) (2014).

[29] A. Volya, Physics of unstable nuclei: from structure to sequential decays, EPJ Web of Conf. 38, 03003 (2012).
[30] F. Rotaru, F. Negoita, S. Grévy, J. Mrazek, S. Lukyanov, F. Nowacki, A. Poves, O. Sorlin, C. Borcea, R. Borcea, A. Buta, L. Cáceres, S. Calinescu, R. Chevrier, Z. Dombrádi, J. M. Daugas, D. Lebhertz, Y. Penionzhkevich, C. Petrone, D. Sohler, M. Stanoiu, and J. C. Thomas, Unveiling the Intruder Deformed $0_{2}^{+}$State in ${ }^{34} \mathrm{Si}$, Phys. Rev. Lett. 109, 092503 (2012).

[31] J. Wood, K. Heyde, W. Nazarewicz, M. Huyse, and P. van Duppen, Coexistence in even-mass nuclei, Phys. Rep. 215, 101 (1992).

[32] J. W. Olness, W. R. Harris, A. Gallmann, F. Jundt, D. E. Alburger, and D. H. Wilkinson, Levels of $\mathrm{S}^{36}$ from $\mathrm{S}^{34}(t, p) \mathrm{S}^{36}$, Phys. Rev. C 3, 2323 (1971).

[33] E. Flynn, O. Hansen, R. Casten, J. Garrett, and F. AjzenbergSelove, The $(t, p)$ reaction on ${ }^{36,38,40} \mathrm{Ar}$, Nucl. Phys. A 246, 117 (1975).

[34] http://amdc.impcas.ac.cn/web/masseval.html.

[35] P. Doornenbal, H. Scheit, S. Takeuchi, Y. Utsuno, N. Aoi, K. Li, M. Matsushita, D. Steppenbeck, H. Wang, H. Baba, E. Ideguchi, N. Kobayashi, Y. Kondo, J. Lee, S. Michimasa, T. Motobayashi, T. Otsuka, H. Sakurai, M. Takechi, Y. Togano, and K. Yoneda, Low $-z$ shore of the "island of inversion" and the reduced neutron magicity toward ${ }^{28}$ O, Phys. Rev. C 95, 041301(R) (2017).

[36] E. K. Warburton, D. E. Alburger, J. A. Becker, B. A. Brown, and S. Raman, Probe of the shell crossing at $A=40$ via beta decay: Experiment and theory, Phys. Rev. C 34, 1031 (1986).

[37] B. Abromeit, S. L. Tabor, V. Tripathi, R. S. Lubna, D. Caussyn, R. Dungan, K. Kravvaris, E. Rubino, P.-L. Tai, and A. Volya, High spin structure of ${ }^{39} \mathrm{Ar}$ and the FSU cross-shell interaction, Phys. Rev. C 100, 014310 (2019).

[38] E. Rivet, R. H. Pehl, J. Cerny, and B. G. Harvey, Investigation of high spin levels preferentially populated by the $(\alpha, d)$ reaction, Phys. Rev. 141, 1021 (1966).

[39] R. D. Vecchio, R. Kouzes, and R. Sherr, Experimental study of $\left(\mathrm{f}_{72}{ }^{2}\right) 7^{+}$states in s-d shell nuclei, Nucl. Phys. A 265, 220 (1976).

[40] R. H. Pehl, E. Rivet, J. Cerny, and B. G. Harvey, $\mathrm{C}^{12}(\alpha, d) \mathrm{N}^{14}$ reaction, Phys. Rev. 137, B114 (1965).

[41] C. C. Lu, M. S. Zisman, and B. G. Harvey, High-spin states of configuration $\left(1 d_{\frac{5}{2}}\right)_{5+, 0}{ }^{2}$ and $\left(1 g_{\frac{9}{2}}\right)_{9+, 0}{ }^{2}$ strongly populated by the $(\alpha, d)$ reaction, Phys. Rev. 186, 1086 (1969).

[42] A. V. D. Woude and R. D. Meijer, Two-nucleon $t=0$ transfer reactions in the 0p shell, Nucl. Phys. A 258, 199 (1976).

[43] B. Harvey, J. Cerny, R. Pehl, and E. Rivet, Levels involving $\mathrm{A}\left(d_{52}\right)_{5}{ }^{2}$ state in light nuclei, Nucl. Phys. 39, 160 (1962).

[44] B. G. Harvey and J. Cerny, Reaction $\mathrm{C}^{12}(\alpha, d) \mathrm{N}^{14}$, Phys. Rev. 120, 2162 (1960).

[45] W. A. Richter, B. A. Brown, R. Longland, C. Wrede, P. Denissenkov, C. Fry, F. Herwig, D. Kurtulgil, M. Pignatari, and $\mathrm{R}$. Reifarth, Shell-model studies of the astrophysical $r p$-process reactions ${ }^{34} \mathrm{~S}(p, \gamma){ }^{35} \mathrm{Cl}$ and ${ }^{34 g, m} \mathrm{Cl}(p, \gamma){ }^{35} \mathrm{Ar}$, Phys. Rev. C 102, 025801 (2020). 